\title{
Collisionless evaporation from cluster elliptical galaxies
}

\section{A contributor to the intracluster stellar population}

\author{
V. Muccione ${ }^{1}$ and L. Ciotti ${ }^{2}$ \\ 1 Observatoire de Genève, chemin des Maillettes 51, 1290 Sauverny, Suisse \\ 2 Dipartimento di Astronomia, Università di Bologna, via Ranzani 1, 40127 Bologna, Italy \\ e-mail: luca.ciotti@unibo.it
}

Received 22 December 2003 / Accepted 3 April 2004

\begin{abstract}
By means of simple numerical models we discuss whether collisionless stellar evaporation from cluster elliptical galaxies could be an effective mechanism for the production of intracluster stellar populations. The effectiveness of this mechanism is due to the fact that, for realistic galaxy and cluster models, the galaxy oscillation periods near equilibrium configurations in the cluster tidal field are of the same order of stellar orbital times in the external parts of the galaxies themselves. With the aid of Monte-Carlo simulations we explore the evolution of stellar orbits in oscillating galaxies placed near different equilibrium positions. We found that, over an Hubble time, the main effect is a substantial expansion of the galactic outskirts, particularly affecting the galaxy at the cluster center and those orbiting near the cluster core radius: overall, approximately the $10 \%$ of the galaxy mass is affected. Thus, the proposed mechanism could be of some importance in the shaping of the halo of cD galaxies and in making easier "galaxy harassment" in the formation of the intracluster stellar population.
\end{abstract}

Key words. galaxies: elliptical and lenticular, cD - Galaxy: evolution - galaxies: interactions galaxies: kinematics and dynamics

\section{Introduction}

Observational evidences of an intracluster stellar population (hereafter ISP) are mainly based on the detection of planetary nebulae and red giant branch stars freely floating in the intergalactic space. For example, Theuns \& Warren (1996) identified 10 intergalactic planetary nebulae in the Fornax cluster, while Méndez et al. (1997) detected 11 intergalactic objects in the Virgo cluster whose cumulative luminosity functions is in good agreement with planetary nebula luminosity function. In addition, Ferguson et al. (1998) identified an intergalactic red giant branch stars population in the Virgo cluster, while Feldmeier et al. (1998) (with observations of three blank fields in the Virgo cluster), confirmed that a significant fraction of Virgo's starlight is due to the ISP. More recently Okamura et al. (2002) have identified 38 candidates of intracluster planetary nebulae in the core of the Virgo cluster. Overall, the data suggest that approximately $10 \%$ (or even more) of the stellar mass of the cluster is in intergalactic stars (e.g., see Ferguson et al. 1998; Arnaboldi et al. 2002; Durrel et al. 2002; Arnaboldi et al. 2003; Totani 2003; see also Arnaboldi et al. 2003, and references therein).

Send offprint requests to: V. Muccione, e-mail: veruska.muccione@obs. unige.ch
The usual scenario assumed to explain the finding above is that the tidal interactions between galaxies (for example during a fast encounter, e.g., see Merritt 1983, 1984, 1985), and of galaxies with the cluster gravitational field, lead to a substantial stripping of stars from galaxies to the parent cluster (the so-called "galaxy harassment" scenario, see, e.g., Moore et al. 1996; Napolitano et al. 2003). In this paper we explore an additional "stripping" mechanism, namely we quantitatively discuss the effect of resonant interaction between stellar orbits inside the galaxies and the cluster tidal field (hereafter, CTF). The present study is supported by the fact that the characteristic oscillation times of a galaxy near its equilibrium position in the CTF and the mean stellar orbital times in the galaxy external regions are of the same order of magnitude, as already recognized by Ciotti \& Giampieri (1998, hereafter CG98).

In fact, Hawley \& Peebles (1975) reported a possible indication (confirmed by Thompson 1976), that the galaxies are preferentially aligned along the radius vector to the center of the cluster for the Coma cluster; they also suggested the CTF as the possible cause of the alignment. The best evidence for alignment is for brightest cluster galaxies: Adams et al. (1980) found, in 7 very elongated clusters, a general trend for ellipticals to be aligned with the cluster major axis, and also Trevese et al. (1992) found a strong alignment of the brightest 
galaxy major with the long axis of the parent cluster. Numerical $N$-body simulations (Ciotti \& Dutta 1994, hereafter CD94) indeed confirmed the hypothesis that the CTF could be at the origin of the observed alignment and, in particular, revealed that their model elliptical galaxies (Es) behaved with good approximation as rigid bodies. In a complementary analytical exploration of this problem CG98 determined the equilibrium positions of triaxial ellipsoids in the CTF for various cases, and showed that the oscillations period of the galaxies, when slightly displaced from their equilibrium configurations, are of the same order of magnitude of the stellar orbital periods in the galaxy outskirts. This curious finding naturally leads to ask what could be the effect of possible "resonances" between stellar orbital periods in cluster Es and their oscillation periods. Unfortunately, the $N$-body simulations of CD94 were characterized by a limited number of particles (i.e., $N \simeq 3 \times 10^{4}$ ) and by the usual softening, so that evaporations rates could not be properly investigated.

In order to investigate the scenario described above, Muccione \& Ciotti (2003a,b, hereafter MC03a,b) performed a few preliminary Monte-Carlo simulations of orbital exploration of oscillating galaxies, and the results were encouraging, with estimated escape rates summing up to 10 per cent of the initial galaxy mass over an Hubble time. In the present paper we considerably extend these two previous investigations, by assuming that the galaxies are triaxial ellipsoids and two cases are considered: when the center of mass of the ellipsoid is at rest at the equilibrium point of the field generated by the cluster, and when it is placed on a circular orbit around the center of a spherically symmetric cluster. After deriving the equations of the motion of a star inside an oscillating galaxy, we integrate numerically these equations under different initial conditions realized by using a Monte-Carlo extraction.

The paper is organized as follows. In Sect. 2 we briefly review the proper physical setting of the problem, and in Sect. 3 we describe the adopted galaxy and cluster models. In Sect. 4 we describe in the numerical integration scheme, while in Sect. 5 we present the main results, that are finally summarized and discussed in Sect. 6. In the Appendix, the specific technique adopted to obtain the gravitational field inside the galaxy models and other useful dynamical quantities are briefly described.

\section{The physical setting}

\subsection{Triaxial galaxy at the center of a triaxial cluster}

Following the analytical treatment of CG98, we start presenting the simple case of a spinless galaxy with its center of mass at rest at the center of a triaxial galaxy cluster. In CG98 it was shown that the galactic equilibrium configurations correspond to the galaxy inertia ellipsoid oriented along the CTF principal directions: without loss of generality we assume that in the (inertial) Cartesian coordinate system $C$, the CTF tensor $\boldsymbol{T}$ is in diagonal form, with components $T_{i}(i=1,2,3)$.

By using three successive counterclockwise rotations $(\varphi$ around $x$ axis, $\vartheta$ around $y^{\prime}$ axis and $\psi$ around $z^{\prime \prime}$ axis), CG98 showed that the linearized equations of motion for the galaxy near the equilibrium configurations can be written as

$\left\{\begin{array}{l}\ddot{\varphi}=\frac{\Delta T_{32} \Delta I_{32}}{I_{1}} \varphi, \\ \ddot{\vartheta}=\frac{\Delta T_{31} \Delta I_{31}}{I_{2}} \vartheta, \\ \ddot{\psi}=\frac{\Delta T_{21} \Delta I_{21}}{I_{3}} \psi,\end{array}\right.$

where $\Delta T_{i j} \equiv T_{i}-T_{j}$, and $I_{i}$ are the principal components of the galaxy inertia tensor. If we also assume that $T_{1} \geq T_{2} \geq T_{3}$ and $I_{1} \leq I_{2} \leq I_{3}$, then the equilibrium position of Eq. (1) is stable (for the geometrical interpretation of this condition see Sect. 3), and the solution of Eq. (1) is

$\varphi=\varphi_{\mathrm{M}} \cos \left(\omega_{\varphi} t\right), \vartheta=\vartheta_{\mathrm{M}} \cos \left(\omega_{\vartheta} t\right), \psi=\psi_{\mathrm{M}} \cos \left(\omega_{\psi} t\right)$,

where

$\left\{\begin{array}{l}\omega_{\varphi}=\sqrt{\frac{\Delta T_{23} \Delta I_{32}}{I_{1}}}, \\ \omega_{\vartheta}=\sqrt{\frac{\Delta T_{13} \Delta I_{31}}{I_{2}}}, \\ \omega_{\psi}=\sqrt{\frac{\Delta T_{12} \Delta I_{21}}{I_{3}}},\end{array}\right.$

are the three independent oscillations frequencies. For simplicity we assume that at $t=0$ the galaxy is at the maximum deviation from the equilibrium configuration (with null angular velocity).

For computational reasons the best reference system in which calculate and discuss the stellar orbits is the oscillating reference system $C^{\prime}$ in which the galaxy is at rest, and its inertia tensor is in diagonal form. As a consequence of this choice, the equation of the motion for a galactic star in $C^{\prime}$ is

$\ddot{\boldsymbol{\xi}}=\mathcal{R}^{\mathrm{T}} \ddot{\boldsymbol{x}}-2 \boldsymbol{\Omega} \wedge \boldsymbol{v}-\dot{\boldsymbol{\Omega}} \wedge \boldsymbol{\xi}-\boldsymbol{\Omega} \wedge(\boldsymbol{\Omega} \wedge \boldsymbol{\xi})$,

where $\xi$ and $v=\dot{\xi}$ are the stellar position and velocity in $C^{\prime}$, the suffix " $T$ " means transpose, and $\mathcal{R}$ is the orthogonal transformation matrix between $C$ and $C^{\prime}$ so that $\boldsymbol{x}=\mathcal{R} \boldsymbol{\xi}$. The explicit form of $\mathcal{R}$ is given by Eq. (9) in CG98: as well known, this matrix define the angular velocity of $C^{\prime}$ that, expressed in $C^{\prime}$, reads

$$
\begin{aligned}
\boldsymbol{\Omega}= & (\dot{\varphi} \cos \vartheta \cos \psi+\dot{\vartheta} \sin \psi, \\
& -\dot{\varphi} \cos \vartheta \sin \psi+\dot{\vartheta} \cos \psi, \dot{\varphi} \sin \vartheta+\dot{\psi}) .
\end{aligned}
$$

If we indicate with $\phi_{\mathrm{g}}=\phi_{\mathrm{g}}(\boldsymbol{\xi})$ the galactic gravitational potential in $C^{\prime}$, then in Eq. (4)

$\mathcal{R}^{\mathrm{T}} \ddot{\boldsymbol{x}} \equiv-\nabla_{\boldsymbol{\xi}} \phi_{\mathrm{g}}+\mathcal{R}^{\mathrm{T}} \boldsymbol{T} \mathcal{R} \boldsymbol{\xi}$,

where $\nabla_{\xi}$ is the gradient operator in $C^{\prime}$, and the direct effect of the cluster gravitational field is obtained in the tidal approximation. In this scheme, each star experience two different influences from the ambient cluster: an indirect one, due to the induced oscillatory motion of the galaxy, and the direct acceleration due to the galaxy and the cluster mass distributions. Of these two last terms, the by far more important within the galaxy is the galactic gravitational field. In Sects. 3 and 4, and in Appendices B and C, we give the explicit expression for the quantities appearing in Eqs. (4) and (6) for the adopted galaxy and cluster models. 


\subsection{Triaxial galaxy on circular orbit in a spherical cluster}

The second, more complicate (but still highly idealized, although more astrophysically relevant) case of interest is represented by a triaxial galaxy with its center of mass in circular orbit of radius $r$ in a plane (say, $z=0$ ) of a spherical cluster, with angular velocity $\boldsymbol{\Omega}_{\mathrm{c}}=\left(0,0, \Omega_{\mathrm{c}}\right)$, where

$\Omega_{\mathrm{c}}^{2}(r)=\frac{G M_{\mathrm{c}}(r)}{r^{3}}$,

and where $M_{\mathrm{c}}(r)$ is the cluster mass within $r$. Following the treatment of CG98, the analogues of Eqs. (1) are

$\left\{\begin{array}{l}\ddot{\varphi}=-\frac{\Omega_{\mathrm{c}}^{2} \Delta I_{32}}{I_{1}} \varphi+\frac{\Omega_{\mathrm{c}}\left(I_{1}-\Delta I_{32}\right)}{I_{1}} \dot{\vartheta}, \\ \ddot{\vartheta}=-\frac{\left(\Omega_{\mathrm{c}}^{2}+\Delta T_{13}\right) \Delta I_{31}}{I_{2}} \vartheta-\frac{\Omega_{\mathrm{c}}\left(I_{2}-\Delta I_{31}\right)}{I_{2}} \dot{\varphi},\end{array}\right.$

while the equation for $\psi$ remains unchanged. The explicit solution of Eqs. (8) is given in Appendix A. In this case there are two different equilibrium configurations: the first corresponds to the galaxy's major axis directed toward the cluster center, and the second to the galaxy major axis perpendicular to the orbital plane (see CG98). For simplicity in this paper we restrict to the first case only.

Due to the system configuration we now need two coordinate transformations in order to obtain the equations of motion for a star in the galaxy. The first transforms the (cluster) inertial system $C$ in the system $C_{\mathrm{c}}$ centered on the galaxy center of mass (rotating with the angular velocity $\boldsymbol{\Omega}_{\mathrm{c}}$ and with its $z$ axis parallel to the $z$ axis of $C$ ), and the second transforms $C_{\mathrm{c}}$ in the system $C^{\prime}$ in which the galaxy is at rest and its inertia tensor is in diagonal form. The relation between the position vector $x$ in $C$ and the position vector $\boldsymbol{\xi}$ in $C^{\prime}$ is given by $\boldsymbol{x}=\boldsymbol{r}+\mathcal{R}^{\prime} \boldsymbol{\xi}$, where $\mathcal{R}^{\prime}=\mathcal{R}_{\mathrm{c}} \mathcal{R}$. $\mathcal{R}$ is the same as in Eq. (4), while

$\mathcal{R}_{\mathrm{c}}=\left(\begin{array}{ccc}\cos \Omega_{\mathrm{c}} t & -\sin \Omega_{\mathrm{c}} t & 0 \\ \sin \Omega_{\mathrm{c}} t & \cos \Omega_{\mathrm{c}} t & 0 \\ 0 & 0 & 1\end{array}\right)$.

Straightforward algebra then shows that the equations of motion for a star as seen from $C^{\prime}$ are similar to Eq. (4), where $\boldsymbol{\Omega}$ is substituted by $\boldsymbol{\Omega}^{\prime}=\boldsymbol{\Omega}+\mathcal{R}^{\mathrm{T}} \boldsymbol{\Omega}_{\mathrm{c}}, \boldsymbol{\Omega}$ is given in Eq. (5), and

$$
\begin{aligned}
\mathcal{R}^{\mathrm{T}} \boldsymbol{\Omega}_{\mathrm{c}}= & (\sin \varphi \sin \psi-\cos \varphi \sin \vartheta \cos \psi, \\
& \sin \varphi \cos \psi+\cos \varphi \sin \vartheta \sin \psi, \cos \varphi \cos \vartheta) \Omega_{\mathrm{c}} .
\end{aligned}
$$

Moreover, $\mathcal{R}^{\mathrm{T}} \ddot{\boldsymbol{x}}$ is substituted by $\mathcal{R}^{\prime \mathrm{T}}(\ddot{\boldsymbol{x}}-\ddot{\boldsymbol{r}}) \simeq-\nabla_{\boldsymbol{\xi}} \phi_{\mathrm{g}}+\mathcal{R}^{\mathrm{T}} \boldsymbol{T} \mathcal{R} \boldsymbol{\xi}$, where now $\boldsymbol{T}$ is the CTF tensor as seen in $C_{\mathrm{c}}$. Note that in tidal approximation, the center of mass of the galaxy will consistently remains in the circular orbit, independently of the galaxy oscillations (see CG98). In Sects. 3 and 4, and in Appendices A, $\mathrm{B}$, and $\mathrm{C}$ we give the explicit expression for the various quantities of interest for the adopted galaxy and cluster models.

\section{Galaxy and cluster models}

We now present the specific galaxy and cluster density distributions adopted in the numerical simulations, and we derive the required explicit expressions for the galaxy inertia tensor and the CTF: a similar approach can be found in Valluri (1993). For simplicity we assume that the galaxy and cluster densities are stratified on homeoids. In MC03ab we adopted triaxial $n=0$ Ferrers (1887) and Hernquist (1990) models, respectively: here we recall the results obtained when adopting the ellipsoidal generalization of the widely used $\gamma$-models (Dehnen 1993):

$\rho_{\mathrm{g}}(m)=\frac{M_{\mathrm{g}}}{\alpha_{1} \alpha_{2} \alpha_{3}} \frac{3-\gamma}{4 \pi} \frac{1}{m^{\gamma}(1+m)^{4-\gamma}}, \quad 0 \leq \gamma<3$,

while we perform the extended exploration of the parameter space by using the density distribution

$\rho_{\mathrm{g}}(m)=\frac{M_{\mathrm{g}}}{\alpha_{1} \alpha_{2} \alpha_{3}} \frac{15}{2 \pi} \frac{1}{(1+m)^{6}}$,

where $M_{\mathrm{g}}$ is the total mass of the galaxy, and

$m^{2}=\sum_{i=1}^{3} \frac{\xi_{i}^{2}}{\alpha_{i}^{2}}, \quad \alpha_{1} \geq \alpha_{2} \geq \alpha_{3}$.

Quite obviously, the density profiles in Eq. (11) are more realistic than that in Eq. (12): however, for technical reasons described in Appendix $\mathrm{C}$, this latter density profile allows for much more faster numerical simulations (i.e., a larger number of particles). Fortunately, the obtained escape rates in all the explored models, here and in MC03ab, are remarkably similar, and so can be considered quite robust (within the considered scenario).

The inertia tensor of generic homeoids are given by

$I_{i}=\frac{4 \pi}{3} \alpha_{1} \alpha_{2} \alpha_{3}\left(\alpha_{j}^{2}+\alpha_{k}^{2}\right) h_{\mathrm{g}}$

where $h_{\mathrm{g}}=\int_{0}^{\infty} \rho_{\mathrm{g}}(m) m^{4} \mathrm{~d} m$, and so the stability requirement $I_{1} \leq I_{2} \leq I_{3}$ is satisfied. Note also that, according to Eq. (3), the frequencies for homeoidal stratifications do not depend on the specific density distribution assumed, but only on the quantities $\left(\alpha_{1}, \alpha_{2}, \alpha_{3}\right)$. For a better comparison with observations, in the following we use the two ellipticities ${ }^{1}$

$\frac{\alpha_{2}}{\alpha_{1}} \equiv 1-\epsilon, \quad \frac{\alpha_{3}}{\alpha_{1}} \equiv 1-\eta, \quad \epsilon \leq \eta \leq 1$.

A rough estimate of characteristic stellar orbital times inside $m$ for an homeoidal distribution is given by $P_{\text {orb }}(m) \simeq 4 P_{\text {dyn }}(m)=$ $\sqrt{3 \pi / G \overline{\rho_{\mathrm{g}}}(m)}$, where for a $\gamma$ model the mean galaxy density inside $m$ is

$\bar{\rho}_{\mathrm{g}}(m)=\frac{3 M_{\mathrm{g}}}{4 \pi \alpha_{1} \alpha_{2} \alpha_{3}} \frac{1}{m^{\gamma}(1+m)^{3-\gamma}}$,

and the galaxy mass inside $m$ is given by:

$M_{\mathrm{g}}(m)=4 \pi \alpha_{1} \alpha_{2} \alpha_{3} \int_{0}^{m} \rho_{\mathrm{g}}(t) t^{2} \mathrm{~d} t=M_{\mathrm{g}} \frac{m^{3-\gamma}}{(1+m)^{3-\gamma}}$.

\footnotetext{
${ }^{1}$ In fact, it can be easily proved the for generic homeoidal distributions, $\epsilon$ and $\eta$ defined according to Eq. (15) are the true ellipticities in the relative projection planes.
} 
Thus,

$$
\begin{aligned}
P_{\mathrm{orb}}(m) \simeq & 9.35 \times 10^{6} \sqrt{\frac{\alpha_{1,1}^{3}(1-\epsilon)(1-\eta)}{M_{\mathrm{g}, 11}}} \\
& \times m^{\gamma / 2}(1+m)^{(3-\gamma) / 2} \mathrm{yrs},
\end{aligned}
$$

where $M_{\mathrm{g}, 11} \equiv M_{\mathrm{g}} / 10^{11} M_{\odot}$ and $\alpha_{1,1} \equiv \alpha_{1} / \mathrm{kpc}$ is the galaxy "core" major axis" ${ }^{2}$ thus, in the outskirts of normal galaxies orbital times well exceeds $10^{8}$ or even $10^{9}$ yrs. A similar conclusion is reached also for the model in Eq. (12).

To describe the cluster density distribution we use the simple mass profile

$\rho_{\mathrm{c}}(m)=\frac{\rho_{\mathrm{c} 0}}{\left(1+m^{2}\right)^{2}}$,

where $m$ is given by an identity similar to Eq. (13), with $a_{1} \geq$ $a_{2} \geq a_{3}$; in the case of the spherical cluster we assume $a_{1}=$ $a_{2}=a_{3}$. In the two following Sections we will compute the CTF components associated with Eq. (19).

\subsection{Galaxy at the cluster center}

In Appendix B it is shown that the tidal field components at the center of a non-singular homeoidal distribution (such as that in Eq. (19)), are given by

$T_{i}=-2 \pi G \rho_{\mathrm{c} 0} w_{i}$.

Note that the quantities $w_{i}$ do not depend on the specific density profile, and that $w_{1} \leq w_{2} \leq w_{3}$ for $a_{1} \geq a_{2} \geq a_{3}$, thus fulfilling the conditions for stable equilibrium in Eq. (1). In analogy with definitions (15), we introduce the two cluster ellipticities, $a_{2} / a_{1} \equiv 1-\mu$ and $a_{3} / a_{1} \equiv 1-v$, with $\mu \leq v \leq 1$, and the expansion of the quantities $w_{i}$ for small flattenings is given in Appendix C.

In order to determine the galaxy oscillation frequencies we need, according to Eqs. (3) and (20), to have a realistic estimate of $\rho_{\mathrm{c} 0}$. Unfortunately this quantity is not well measured in real clusters, and for its determination we use the virial theorem, $M_{\mathrm{c}} \sigma_{\mathrm{V}}^{2}=-U$ ( $U$ is the cluster gravitational potential energy and $\sigma_{\mathrm{V}}^{2}$ is the virial velocity dispersion, that we assume to be estimated by the observed velocity dispersion of galaxies in the cluster). The explicit calculations of the factor $2 \pi G \rho_{\mathrm{c} 0}$ is presented in Appendix B.

We now compare the galactic oscillation periods $P_{\varphi}=$ $2 \pi / \omega_{\varphi}, P_{\vartheta}=2 \pi / \omega_{\vartheta}$, and $P_{\psi}=2 \pi / \omega_{\psi}$, with the characteristic stellar orbital times $P_{\text {orb }}$ in galaxies: for simplicity we give here the expansions for small cluster and galactic flattenings. From Eqs. (3), (14), (20), and using Eqs. (C.8)-(C.12) we finally obtains to the leading order in the flattenings

$P_{\varphi} \simeq \frac{8.58 \times 10^{8}}{\sqrt{(v-\mu)(\eta-\epsilon)}} \frac{a_{1,250}}{\sigma_{\mathrm{V}, 1000}} \quad \mathrm{yrs}$,

$P_{\vartheta} \simeq \frac{8.58 \times 10^{8}}{\sqrt{v \eta}} \frac{a_{1,250}}{\sigma_{\mathrm{V}, 1000}} \quad \mathrm{yrs}$,

\footnotetext{
2 We recall here that for the spherically symmetric Hernquist model, (i.e., $\alpha_{1}=\alpha_{2}=\alpha_{3}$ in Eq. (12)), $R_{\mathrm{e}} \simeq 1.8 \alpha_{1}$, while for the density distribution in Eq. (12) $R_{\mathrm{e}} \simeq 0.75 \alpha_{1}$.
}

$P_{\psi} \simeq \frac{8.58 \times 10^{8}}{\sqrt{\mu \epsilon}} \frac{a_{1,250}}{\sigma_{\mathrm{V}, 1000}} \quad \mathrm{yrs}$

where we normalized $\sigma_{\mathrm{V}}$ to $1000 \mathrm{~km} \mathrm{~s}^{-1}$ and $a_{1}$ to $250 \mathrm{kpc}$. So, a comparison of quantities above with Eq. (18) shows that in the outer halo of giant Es the stellar orbital times can be of the same order of magnitude as the oscillatory periods of the galaxies themselves. For example, in a relatively small (Hernquist) galaxy of $M_{\mathrm{g}, 11}=0.1$ and $\alpha_{1,1}=1, P_{\text {orb }} \simeq 1$ Gyr at $m \simeq 10$ (i.e., at $\simeq 5 R_{\mathrm{e}}$ ), while for a galaxy with $M_{\mathrm{g}, 11}=1$ and $\alpha_{1,1}=3$ the same orbital time is at $m \simeq 7$ (i.e., at $\simeq 3.5 R_{\mathrm{e}}$ ).

\subsection{Galaxy in circular orbit}

In the case of spherical cluster, the CTF tensor in the reference system $C_{\mathrm{c}}$ introduced in Sect. 2.2 is

$\boldsymbol{T}=-\Omega_{\mathrm{c}}^{2}(r)\left(\begin{array}{ccc}3 q-2 & 0 & 0 \\ 0 & 1 & 0 \\ 0 & 0 & 1\end{array}\right)$

where $q(r) \equiv \rho_{\mathrm{c}}(r) / \overline{\rho_{\mathrm{c}}}(r)$ and $\overline{\rho_{\mathrm{c}}} \equiv 3 M_{\mathrm{c}}(r) / 4 \pi r^{3}$ (see, e.g., CD94, CG98). From Eq. (19) we obtain

$M_{\mathrm{c}}(r)=2 \pi a_{1}^{3} \rho_{\mathrm{c} 0}\left(\arctan \tilde{r}-\frac{\tilde{r}}{1+\tilde{r}^{2}}\right)$,

$\bar{\rho}_{\mathrm{c}}(r)=\frac{3 \rho_{\mathrm{c} 0}}{2 \tilde{r}^{3}}\left(\arctan \tilde{r}-\frac{\tilde{r}}{1+\tilde{r}^{2}}\right)$,

$q(r)=\frac{2 \tilde{r}^{3}}{3\left(1+\tilde{r}^{2}\right)^{2}}\left(\arctan \tilde{r}-\frac{\tilde{r}}{1+\tilde{r}^{2}}\right)^{-1}$,

where $\tilde{r} \equiv r / a_{1}$. In analogy with Sect. 3.1, we now compare the galaxy oscillation periods with the mean stellar orbital times, and for simplicity we restrict to the special case of the galaxy oscillating around the $\xi_{3}$-axis only, i.e., we fix $\varphi=0$ and $\vartheta=0$ in Eq. (10). Thus, from the results of Sect. 2.2, and from Eq. (24)

$P_{\psi} \equiv \frac{2 \pi}{\omega_{\psi}} \simeq \frac{2 \pi}{\Omega_{\mathrm{c}}(r)} \frac{1}{\sqrt{3 \epsilon[1-q(r)]}}$,

where we used the last of Eqs. (3) and the expansion for small flattenings of the coefficient involving the galaxy moments of inertia. In Fig. 1 we plot $P_{\psi}$ as a function of $r$ for the two spherical cluster models used in the simulations described in Sect. 5.2, in the cases of an E2 and and E4 galaxy; note that $P_{\psi}$ decreases at increasing galaxy flattening, while its radial trend is non monotonic, with a minimum at $r \simeq a_{1}$, the cluster core radius. The peculiarity of this location has been already noted (e.g., see CD94), and its impact on orbital evolution will be evident in Sect. 5.2.

\section{The numerical integration scheme}

\subsection{Dimensionless equations}

In the numerical simulations we use as time normalization the Hubble time $t_{\mathrm{H}}=15$ Gyrs, as mass normalization the galaxy 


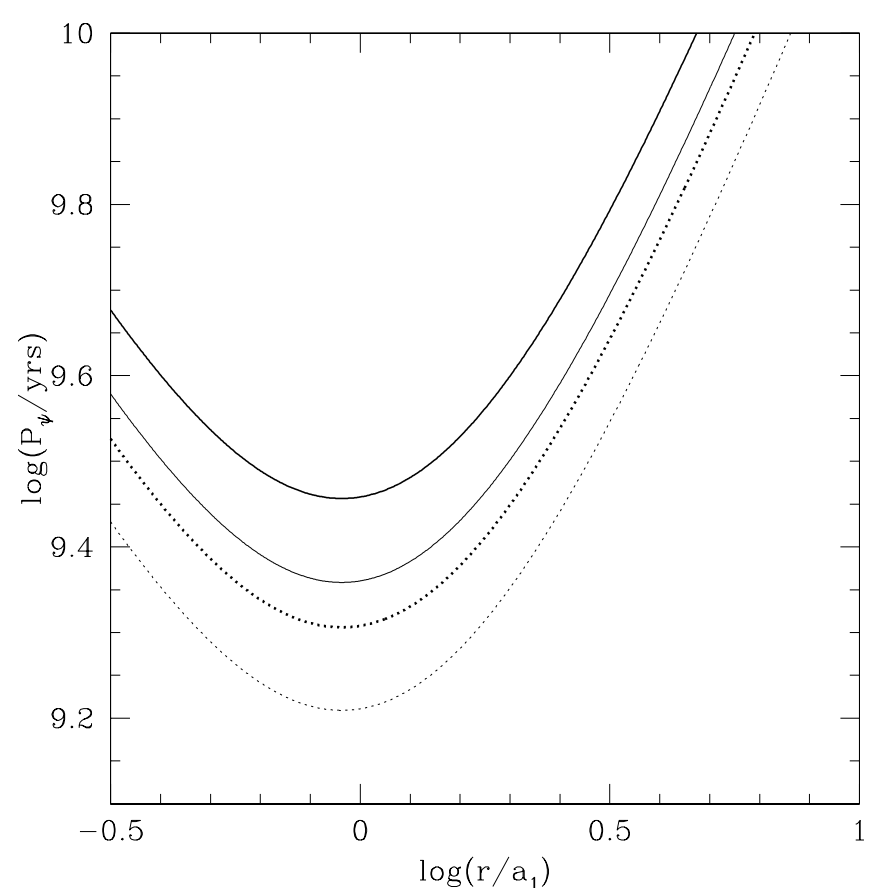

Fig. 1. The oscillation period around the $\xi_{3} \| \boldsymbol{\Omega}_{\mathrm{c}}$ axis for a galaxy in circular orbit of radius $r$ in a cluster of core radius $a_{1}$ and velocity dispersion $\sigma_{\mathrm{V}}$. Solid lines refer to an E2 galaxy, while dotted lines to an $\mathrm{E} 4$ galaxy. Cluster model $\mathrm{Ca}$ (heavy lines) is characterized by $a_{1}=250 \mathrm{kpc}$ and $\sigma_{\mathrm{V}}=1000 \mathrm{~km} \mathrm{~s}^{-1}$, while model Cb by $a_{1}=100 \mathrm{kpc}$ and $\sigma_{\mathrm{V}}=500 \mathrm{~km} \mathrm{~s}^{-1}$.

mass $M_{\mathrm{g}}$, and as length normalization $\alpha_{1}$, the homeoidal scalelength corresponding to the galaxy major axis. Accordingly, Eq. (4) in dimensionless form reads

$$
\begin{aligned}
\frac{\mathrm{d}^{2} \tilde{\boldsymbol{\xi}}}{\mathrm{d} \tau^{2}}= & -\frac{G M_{\mathrm{g}} t_{\mathrm{H}}^{2}}{\alpha_{1}^{3}} \nabla_{\tilde{\boldsymbol{\xi}}} \tilde{\phi}_{\mathrm{g}} \\
& -2 \omega_{\mathrm{c}} t_{\mathrm{H}} \tilde{\boldsymbol{\Omega}} \wedge \tilde{\boldsymbol{v}}-\omega_{\mathrm{c}}^{2} t_{\mathrm{H}}^{2}[\dot{\tilde{\mathbf{\Omega}}} \wedge \tilde{\boldsymbol{\xi}}+\tilde{\boldsymbol{\Omega}} \wedge(\tilde{\mathbf{\Omega}} \wedge \tilde{\boldsymbol{\xi}})],
\end{aligned}
$$

where $\tau \equiv t / t_{\mathrm{H}}$ and $\phi_{\mathrm{g}} \equiv\left(G M_{\mathrm{g}} / \alpha_{1}\right) \tilde{\phi_{\mathrm{g}}}$; note that from Eq. (3) $\omega_{i}=\omega_{\mathrm{c}} \tilde{\omega}_{i}$ where $\omega_{\mathrm{c}} \equiv \sqrt{2 \pi G \rho_{\mathrm{c} 0}}$, and so from Eqs. (2) and (5) $\boldsymbol{\Omega}=\omega_{\mathrm{c}} \tilde{\boldsymbol{\Omega}}$. In addition, consistently with the adopted "small oscillations approximation", the angular velocity is used in linearized form

$\mathbf{\Omega}(\dot{\varphi}, \dot{\vartheta}, \dot{\psi})$

i.e., in Eq. (5) we retained only linear terms in $\varphi_{M}, \vartheta_{M}, \psi_{M}$; note that we neglect the direct effect of the cluster gravitational field on stellar orbits (cf. with Eq. (6)).

According to the discussion in Sect. 2.2, the dimensionless equations of the motion for a star in the galaxy on a circular orbit are similar to Eq. (29), where now $\omega_{\mathrm{c}} \equiv \sqrt{2 \pi G \overline{\rho_{\mathrm{c}}}}$, we again disregard the direct effect of the CTF, $\tilde{\mathbf{\Omega}}$ is substituted by $\widetilde{\mathbf{\Omega}^{\prime}} \equiv \boldsymbol{\Omega}^{\prime} / \omega_{\mathrm{c}}$, and to the linear order

$\mathbf{\Omega}^{\prime} \simeq\left(\dot{\varphi}-\vartheta \Omega_{\mathrm{c}}, \dot{\vartheta}+\varphi \Omega_{\mathrm{c}}, \dot{\psi}+\Omega_{\mathrm{c}}\right)$.

Note that $\Omega_{\mathrm{c}} / \omega_{\mathrm{c}}=\sqrt{2 / 3}$.

\subsection{Initial conditions}

To integrate the second order differential Eqs. (29) describing the motion of a test star in the non-inertial reference frame fixed in the galaxy, we use a code based on an adapted f77, double-precision 4th order Runge-Kutta routine (Press et al. 1986), with adaptive time step. In practice, for a given galaxy and cluster model, we analyzed the behaviour of a large number of orbits $\left(N_{\text {tot }} \sim 10^{4} \div 10^{6}\right)$ over an equivalent Hubble time: from this point of view our simulations are nothing else that a large collection of one-body problems in time dependent potentials. Of course, the initial conditions for each orbit must be realized with some care. First of all, in order to properly sample the galaxy density profile the initial positions are obtained from the Von Neumman rejection method (Press et al. 1986) which uses the (elliptical) cumulative mass distribution $M_{\mathrm{g}}(m) / M_{\mathrm{g}}$ as probability function for the homeoidal radius; the two position angles are then extracted from uniformly populated standard angular intervals (i.e., $0 \leq \varphi<2 \pi$ and $-1 \leq \cos \vartheta \leq 1$ ).

A more delicate issue is represented by the assignment of the three components of the initial velocity. In principle, one should perform a von Neumann rejection method in phasespace, provided the model phase-space distribution function is known at each point. Unfortunately, for triaxial galaxies the only sufficiently general method is the Schwarzschild (1979) orbit linear superposition method, which however for our purposes would be very demanding from the numerical point of view. Here we adopt a more empirical approach, whose consistency is however readily verified. In some simulations, for each initial position in configuration space, we calculate the 1dimensional velocity dispersion $\sigma^{2}$ from the Jeans equations of the spherical analogue of the density distribution in Eqs. (11) and (12), we choose a dimensionless parameter $\chi \leq 1$, and we randomly distribute over the three directions the initial particle velocity so that its square modulus equals $3 \chi \sigma^{2}$. In other simulations, we same procedure was repeated, but assuming a fixed (within a given simulation) fraction $\chi$ of the local escape velocity $v_{\text {esc }}$.

For each simulation (i.e., for assigned galaxy and cluster models, maximum oscillation angles, and $\chi$ value) we actually run the code twice: in the first simulation the galaxy maximum oscillation angles are set to zero (in other words the galaxy is at rest at the equilibrium position in the CTF), and all the initial conditions leading to unacceptable orbits for the unperturbed case are discarded. In this way we can also test the code for energy conservation. In all the computed models the "unperturbed" case pass very well the acceptability test, with relative errors on energy conservation for each orbit never exceeding $\sim 10^{-5}$ over an Hubble time, and without significant spurious evaporation. Thus, we can safely assume that the evaporation rates found in the explored models are a genuine product of galaxy oscillations, and no a product of and inconsistent assignment of the initial conditions.

All the simulations have been performed on GRAVITOR, the Geneva Observatory 132 processors Beowulf cluster ${ }^{3}$ : for

${ }^{3}$ For technical detail on GRAVITOR see http: //obswww. unige. ch/ pfennige/gravitor/gravitor_e.html 
reference, the computation of $10^{4}$ orbits requires $\sim 2 \mathrm{~h}$ when using 10 nodes.

\section{Results}

\subsection{Galaxy at the center of a triaxial cluster}

Here we describe the results of the first of the two cases discussed in Sect. 2, namely the case of a triaxial galaxy at the center of a triaxial cluster. In this case we already have preliminary informations for galaxy models described by Ferrers (1887, see MC03a) and by the more realistic Hernquist (see MC03b) density distribution. In this section we complement these informations with the results obtained when using the density profile in Eq. (12). However in the present case, as for Hernquist models and at variance with Ferrers distribution, the concept of "escape" is quite subtle. In fact, while in truncated galaxy models the escape criterion is obvious, in untruncated models the "escaping" particles must be identified in a different way. In particular we choose to plot, for each test particle in a given model, the ratio $m_{\max } / m_{\mathrm{i}}$ vs. $m_{\mathrm{i}}$, where $m_{\mathrm{i}}$ is the ellipsoidal radius of a given initial condition, while $m_{\max }$ is the maximum ellipsoidal distance reached by that particle over an equivalent Hubble time. We present here only a representative simulation among the several performed, because they remarkably confirm the previous results for Hernquist models described in MC03b. In particular, the shown simulation refers to a galaxy model characterized by the two flattenings $\epsilon=2 / 5$ and $\eta=16 / 25$; the same ellipticities have been also adopted for the cluster, i.e. $\mu=2 / 5$ and $v=16 / 25$. Thus, the adopted galaxy model would be classified, as a function of the observation angle, from an $E 4$ up to an $E 6$ galaxy. Its mass is fixed to $M_{\mathrm{g}}=10^{11} M_{\odot}$, while its core semimajor axis is fixed to $\alpha_{1}=6.58 \mathrm{kpc}$ (corresponding to an effective radius in the spherical model of $\simeq 5 \mathrm{kpc}$ ). The cluster is characterized by $\sigma_{\mathrm{V}}=1000 \mathrm{~km} \mathrm{~s}^{-1}$ and $a_{1}=250 \mathrm{kpc}$. Initial conditions were generated with $m_{\mathrm{i}} \leq 8$ and with a square modulus of the initial velocity of each particle equals to the local $0.3 v_{\text {esc }}^{2}$. The galaxy maximum oscillation angles along the three directions are $\varphi_{\mathrm{M}}=\vartheta_{\mathrm{M}}=\psi_{\mathrm{M}}=0.25$.

The results are shown in Fig. 2. Note how in the unperturbed case, with the adopted $\chi$ value, $m_{\max } / m_{\mathrm{i}}$ increases only slightly, while in the oscillating case this number reaches higher values, of the order of 1.5 or more. These results are very similar to those described in MC03b (see Figs. 1 and 2 there). Note also how the expansion effect is more important in the external galaxy regions (affecting particles with $m_{\mathrm{i}} \gtrsim 3$ ), even though we recall that in the simulations the direct effect of the CTF is not considered: as a consequence, the obtained galaxy expansion is a genuine effect of galactic oscillations. By the way, this is a quite interesting result, that one could relate to the process of $\mathrm{cD}$ formation. In fact, it is well known that it is not easy to grow with galactic cannibalism, at least in numerical simulations, the extended $\mathrm{cD}$ halos (e.g., see Nipoti et al. 2003, and references therein). However, one should recall that the CTF in shallow cores is compressive (e.g., see Valluri 1993, CD94): as a consequence, the final galaxy density profile will be determined by the competing effect of galaxy oscillations and the cluster gravitational field. Thus, in order to
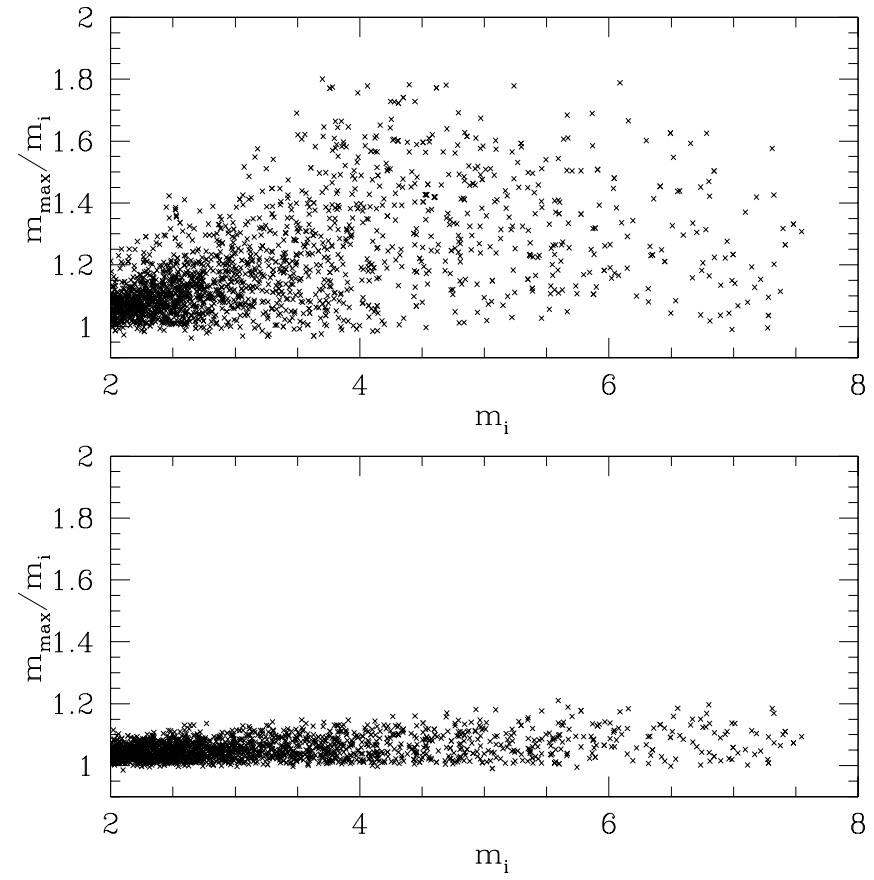

Fig. 2. The ratio $m_{\max } / m_{\mathrm{i}}$ vs. $m_{\mathrm{i}}$ for the subset of particles with $m_{\mathrm{i}} \geq 2$, evaluated over $t_{\mathrm{H}}$. Upper panel: unperturbed galaxy with $\varphi_{\mathrm{M}}=\vartheta_{\mathrm{M}}=$ $\psi_{\mathrm{M}}=0$. Lower panel: the same model with $\varphi_{\mathrm{M}}=\vartheta_{\mathrm{M}}=\psi_{\mathrm{M}}=$ $0.25 \mathrm{rad}$.

quantitatively discuss the possibility that the galaxy sloshing at the cluster center is at the origin of the cD halos, highresolution, self-consistent $N$-body simulations are required.

\subsection{Galaxy on circular orbit in a spherical cluster}

We now move to describe the results of the simulations of galaxies in circular orbit around the cluster center: altough still highly idealized, this cases apply to a larger number of galaxies than the previous one. At variance with the case of a galaxy at the cluster center, galaxies in circular orbit were not studied before, so here we present a larger set of simulations, organized in two groups. In the first set of simulations the spherical cluster is characterized by $\sigma_{\mathrm{V}}=1000 \mathrm{~km} \mathrm{~s}^{-1}$ and $a_{1}=250 \mathrm{kpc}$ (model Ca), while in the second set $\sigma_{\mathrm{V}}=500 \mathrm{~km} \mathrm{~s}^{-1}$ and $a_{1}=100 \mathrm{kpc}$ (model Cb). For each model cluster we study three different positions of the galaxy center of mass, namely circular orbits with $r / a_{1}=0.5$ (models Cai and Cbi), with $r / a_{1}=1$ (models Cac and Cbc), and finally with $r / a_{1}=2$ (models $\mathrm{Cao}$ and $\mathrm{Cbo}$ ). In order to reduce the dimensionality of the parameter space, in all the simulations we fix a galaxy model with $M_{\mathrm{g}}=10^{11} M_{\odot}, \alpha_{1}=7 \mathrm{kpc}, \epsilon=0.2$ and $\eta=0.4$ (thus corresponding to a less flattened galaxy than that used in Sect. 5.1). Finally, we explore different cases of initial velocities of the test particles, with $\chi$ in the range $0.1-0.5$. In all cases, we restrict our analysis to the case of a galaxy oscillating only around the $\xi_{3}$ axis (the axis perpendicular to the orbital plane of the galaxy center of mass), and we adopt $\psi_{\mathrm{M}}=0.2 \mathrm{rad}$.

We note here that the set-up of the initial conditions is considerably more time-expensive that in the case of the galaxy at the cluster center. In fact, in the present case, "galaxy at 

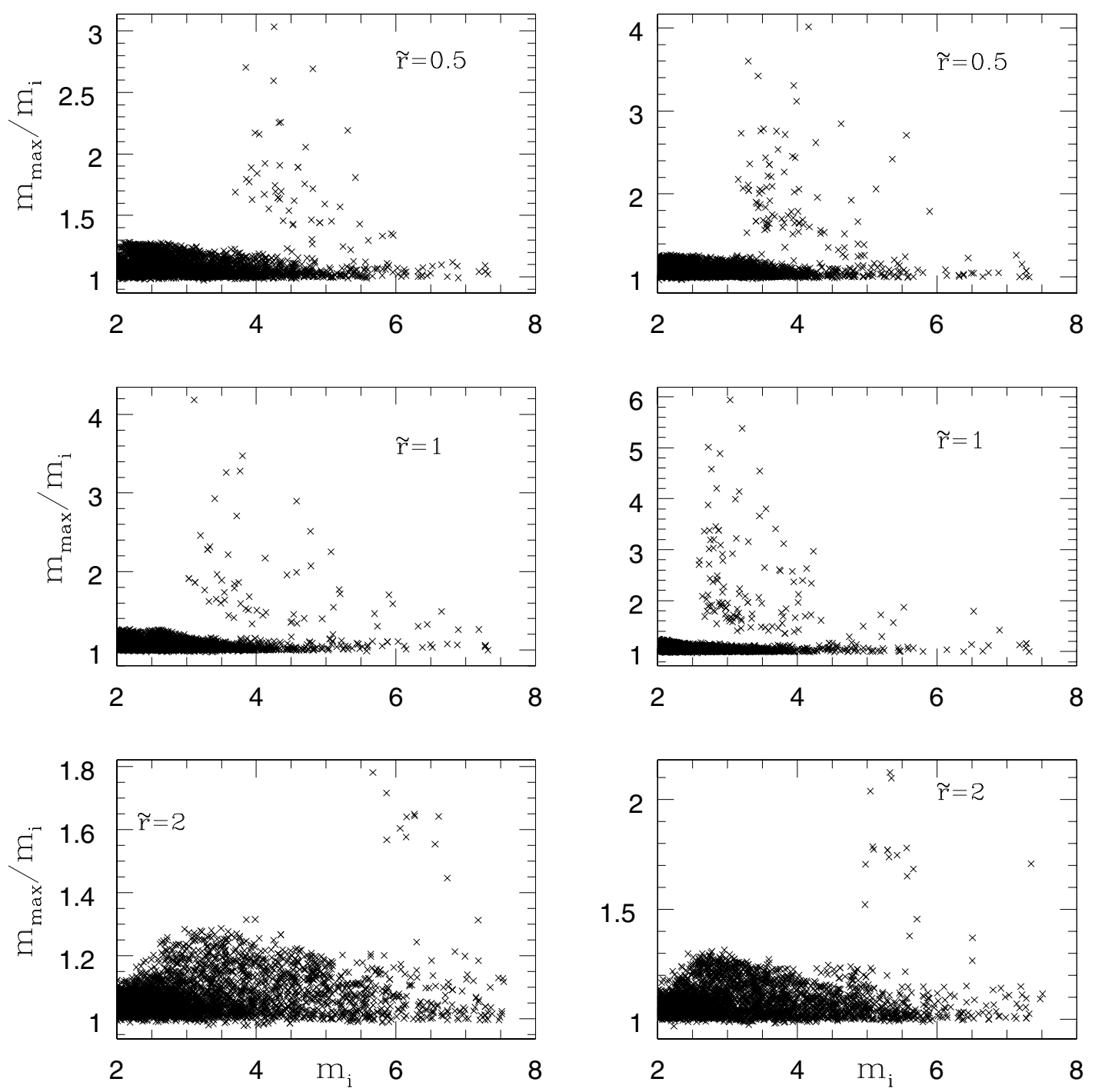

Fig. 3. The ratio $m_{\max } / m_{\mathrm{i}}$ vs. $m_{\mathrm{i}}$ for the subset of particles with $m_{\mathrm{i}} \geq 2$, evaluated over $t_{\mathrm{H}}$, in the case of a galaxies in circular orbit around the cluster center. Left panels: from the top, models Cai, Cac, and Cao, respectively. Right panels: from the top, models Cbi, Cbc, and Cbo, respectively. In all cases the maximum oscillation angle is set to $\psi_{\mathrm{M}}=0.2 \mathrm{rad}$.

rest" means that the galaxy center of mass is actually (uniformly) rotating around the cluster center, and this induces a centrifugal acceleration on the galaxy stars, as apparent by setting $\varphi=\vartheta=\dot{\psi}=0$ in Eq. (31). Thus, if one erroneously adopt as stellar orbit initial conditions the same conditions adopted in the previous section, the result is a vigorous stellar escape, just due to the centrifugal acceleration. Accordingly, test numerical simulations (not shown here) were characterized by a significant number of stars escaped up to $m_{\max } \simeq 100$. Of course this is not a physical process, but only the result of the nonequilibrium initial conditions: in order to avoid this problem we proceeded as follows. For each galaxy model, we first performed the orbital calculation for a large number (say $10^{5}$ ) initial conditions arranged as in Sect. 5.1, maintaining the galaxy in uniform rotation around the cluster center and without oscillations. We then discarded all the initial conditions corresponding to orbits for which, over an Hubble time, $m_{\max } / m_{\mathrm{i}} \gtrsim 1.2$, in order to mimic the result shown in the bottom panel of Fig. 2. We finally used the remaining initial conditions to study the orbital evolution in the corresponding oscillating galaxy. The results are summarized in Fig. 3.

Several interesting comments can be made from inspection of Fig. 3. The first, and more obvious, is that also in the rotating galaxies, oscillations are expected to produce stellar evaporation. However, in the rotating cases we invariably found that the largest $m_{\max } / m_{\mathrm{i}}$ are larger that the corresponding quantity relative to the galaxies at the cluster center (cf. Fig. 2), and this is due to the additional effect of the centrifugal term on escaping stars. Strictly related to this point is also the systematic behavior of $m_{\max } / m_{\mathrm{i}}$ shown in Fig. 3, where it is apparent how the largest ratios systematically decrease for galaxy models placed at $r=a_{1}, a_{1} / 2$ and $2 a_{1}$, respectively. This non-monotonic trend is just a reflection of the same behavior of $P_{\psi}$, shown in Fig. 1: shorter the galaxy oscillation times, larger are the $m_{\max } / m_{\mathrm{i}}$ that can be reached by escaping stars. In this respect, the peculiar dynamical situation of galaxies orbiting near the cluster core radius it is well known: not only there is a change in the topological nature of the CTF there (see, e.g., CD94), but also 
oscillation periods are the shortest (for a given galaxy model). The third comment about Fig. 3 is the common pattern of the radial behavior of the upper envelope of $m_{\max } / m_{\mathrm{i}}$, i.e., a quite well defined, sharp increase followed by a somewhat smoother decline at increasing $m_{\mathrm{i}}$. This behavior basically corresponds to the place in the galaxy where orbital times (see Eq. (18)) become comparable with the oscillation times. Inside this radius stellar orbits react adiabatically to the forcing due to galaxy oscillations (see CD94), while outside galaxy oscillations become effective in changing significantly the stellar orbits. As expected, the effect becomes however smaller and smaller for stellar orbits with increasing orbital times. Finally, note how there are not significant differences between the results in the large $(\mathrm{Ca})$ and small $(\mathrm{Cb})$ clusters: we interpret this as another manifestation of the comparable oscillatory periods in the two cases (see Fig. 1).

\section{Discussion and conclusions}

In this paper we presented a representative selection of numerical models aimed at the study of collisionless evaporation of stars from cluster elliptical galaxies. From a theoretical point of view this possibility was pointed out in CG98, who showed that characteristic oscillation times of elliptical galaxies in near equilibrium configurations in the tidal field of the parent cluster are curiously comparable to the stellar orbital times in the outskirts of the galaxies themselves. From a numerical point of view the present work represents the natural follow-up of two recent preparatory works on the same subject (MC03ab), where we explored the simplest equilibrium configuration (i.e., a galaxy with the center of mass at rest at the center of a triaxial cluster), but we left unaddressed the more complicate (although more astrophysically representative) case of galaxies with their center of mass in rotation around the (spherical) cluster center. We recall that in all the presented simulations the direct effect of the cluster gravitational field on stellar orbits was not included, in order to study the effects of galaxy oscillations only; we also recall that its effect is compressive inside the cluster core radius, while expanding outside (see, e.g., Valluri 1993, CD94).

The main results can be summarized as follows:

- A general analytical framework suitable for the study of orbital evolution of stars in galaxies oscillating around their stable equilibrium positions in the tidal field of the parent cluster is presented. In particular, we analyzed the cases of triaxial galaxies at the center of a triaxial cluster and in circular orbit around the center of a spherical cluster.

- It is shown that, for realistic galaxy and cluster models, galaxy oscillation times can be comparable to characteristic stellar orbital times in the external galactic regions, and thus important effects on stellar orbits are expected there.

- Numerical simulations of galaxiesat the cluster center remarkably confirm the preliminary results presented in MC03ab (where different galaxy models were adopted). In particular, we found that orbits in the external parts of the parent galaxy are affected by galaxy oscillations, with stars outside $m_{\mathrm{i}} \simeq 3 \simeq 2.25 R_{\mathrm{e}}$ nearly doubling the apocenters. Globally, a galaxy mass fraction of the order of $10 \%$ is affected by this process.

- We speculate that the findings above could be related to the formation of $\mathrm{cD}$ galaxies. In fact, it is well known that numerical simulations of galactic cannibalism, although successful at reproducing BCGs, are in general unable to obtain the extended halos of cDs. In this respect, the fact that the external parts of the galaxies are the only ones affected by collisionless evaporation, is a nice property of the explored scenario. Unfortunately, our simulations cannot be used to study the resulting galactic profiles, and high-resolution $N$-body simulations should be performed to study further the matter.

- For galaxies in circular orbits, the effect of collisionless evaporation is enhanced with respect to the previous case, and stars can reach significantly larger distances. This is not due to the direct effect of the CTF, but to galaxy oscillations only. In accordance with our preparatory analysis, we found that the radial trend of galactic oscillation periods at increasing distance from the cluster center is reflected by the location in the galaxy of the expanded region, being the most affected galaxies those orbiting near the cluster core radius. Even in this cases, the affected galaxy mass fraction ranges is of the order of 5\%-10\%, in interesting agreement with observational estimates.

- The natural consequence of the results listed above is that, if collisionless evaporation is of importance in the production of the ISP (as direct contributor and/or help to the stripping phenomena due the direct CTF effect and to galaxy-galaxy encounters, as investigated in detail by Merritt (1983, 1984, 1985)), the density of ISP (when normalized to the local cluster luminosity in galaxies) should be enhanced around the cluster core radius, and rapidly declining at the cluster outskirts, due to the increase of the galaxy oscillation periods with the distance from the cluster center.

We finally conclude by pointing out that both orbital cases explored in this paper are rather exceptional. First of all, we only considered galaxy models that were initially not tumbling nor rotating. In addition, most cluster galaxies neither rest in the cluster center nor move on circular orbits, but they move on elongated orbits with very different pericentric and apocentric distances from the cluster's center; in a triaxial cluster many orbits are boxes and some orbits can be chaotic. These latter cases can be properly investigated only by direct numerical simulation of the stellar motions inside the galaxies, coupled with the numerical integration of the equations of the motion of the galaxies themselves. In addition, a statistically significant galaxy population made by the sum of galaxies with different masses, scale-lengths, and flattenings, should be considered.

Acknowledgements. We would like to thank Magda Arnaboldi, Giuseppe Bertin, Daniel Pfenniger for useful discussions, and the anonymous Referee for helpful comments. V.M. is grateful to Geneva Observatory for allowing the use of their GRAVITOR beowulf cluster. 


\section{Appendix A: Oscillation angles for a triaxial galaxy in circular orbit}

Here we give the explicit solution of eqs. (8) for assigned initial conditions $\left(\varphi_{0}, \dot{\varphi}_{0}\right)$ and $\left(\vartheta_{0}, \dot{\vartheta}_{0}\right)$. As shown in CG98, the two mixed equations of the second order

$\left\{\begin{array}{l}\ddot{\varphi}=-A_{1} \varphi+B_{1} \dot{\vartheta} \\ \ddot{\vartheta}=-A_{2} \vartheta-B_{2} \dot{\varphi}\end{array}\right.$

(where the 4 coefficients $A_{1}, A_{2}, B_{1}, B_{2}$ are given in Eqs. (8)), can be separated in two identical biquadratic equations, each of them involving only one of the two variables $\varphi$ and $\vartheta$. For example, the equation for $\varphi$ can be written as

$\frac{\mathrm{d}^{4} \varphi}{\mathrm{d} t^{4}}+A \frac{\mathrm{d}^{2} \varphi}{\mathrm{d} t^{2}}+B=0$

where

$$
\begin{aligned}
A \equiv & A_{1}+B_{1} B_{2}+A_{2}=\Omega_{\mathrm{c}}^{2} \\
& \times\left[\frac{(u+v-1)^{2}}{u v}+\frac{1-v}{u}+\frac{(4-3 q)(1-u)}{v}\right],
\end{aligned}
$$

$B \equiv A_{1} A_{2}=\Omega_{\mathrm{c}}^{4} \times \frac{(4-3 q)(1-u)(1-v)}{u v}$,

$u \equiv I_{1} / I_{3}, v \equiv I_{2} / I_{3}$, and the functions $\Omega_{\mathrm{c}}(r)$ and $q(r)$ are given in Sect. 3.2. The standard substitution $\varphi=X \exp (i \omega t)$ in Eq. (A.2) leads to the characteristic equation $\omega^{4}-A \omega^{2}+B=0$, whose solutions can be written as

$\omega_{ \pm} \equiv \sqrt{\lambda_{ \pm}} ; \quad \lambda_{ \pm}=\frac{A \pm \sqrt{A^{2}-4 B}}{2} ;$

note that in case of a stable equilibrium for the galaxy configuration (as assumed in this paper), the roots $\lambda_{ \pm}$are positive, and that from Eqs. (A.3) and (A.4) $\omega \propto \Omega_{\mathrm{c}}$. Thus, the general solution of Eqs. (8) can be written as

$\left\{\begin{array}{l}\varphi=a \cos \left(\omega_{-} t+a_{0}\right)+b \cos \left(\omega_{+} t+b_{0}\right) \\ \vartheta=c \cos \left(\omega_{-} t+c_{0}\right)+d \cos \left(\omega_{+} t+d_{0}\right)\end{array}\right.$

where the four amplitudes $a, b, c, d$ and phases $a_{0}, b_{0}, c_{0}, d_{0}$ must be determined by imposing the 4 initial conditions at $t=0$. The remaining four constraints are provided by the request that Eqs. (A.6) satisfy Eqs. (A.1) at any time:

$\left\{\begin{array}{l}c_{0}=a_{0}+\pi / 2, \\ d_{0}=b_{0}+\pi / 2 ; \\ a\left(\omega_{-}^{2}-A\right)-c B_{1} \omega_{-}=0, \\ b\left(\omega_{+}^{2}-A\right)-d B_{1} \omega_{+}=0:\end{array}\right.$

the identities involving amplitudes can be also written in terms of $C$ and $B_{2}$ instead of $A$ and $B_{1}$. We can now proceed to impose the initial conditions on Eqs. (A.6). The easiest way is to define the 6 new quantities

$$
\left\{\begin{array}{l}
r_{-} \equiv a / c=B_{1} \omega_{-} /\left(\omega_{-}^{2}-A\right), \\
r_{+} \equiv b / d=B_{1} \omega_{+} /\left(\omega_{+}^{2}-A\right), \\
X=\cos a_{0}, \quad Y=\cos b_{0}, \\
U=\sin a_{0}, \quad V=\sin b_{0}:
\end{array}\right.
$$

note that $r_{+}$and $r_{-}$are known. Evaluating Eqs. (A.6) at $t=0$, and using Eqs. (A.7) and (A.8) one obtains

$$
\left\{\begin{array}{l}
r_{-} c X+r_{+} d Y=\varphi_{0}, \quad \omega_{-} c X+\omega_{+} d Y=-\dot{\vartheta}_{0}, \\
c U+d V=-\vartheta_{0}, \quad \omega_{-} r_{-} c U+\omega_{+} r_{+} d V=-\dot{\varphi}_{0}, \\
X^{2}+U^{2}=1, \quad Y^{2}+V^{2}=1
\end{array}\right.
$$

The first 4 equations above can be solved for the unknowns $c X, d Y, c U, d V$ in terms of $r_{ \pm},\left(\vartheta_{0}, \dot{\vartheta}_{0}\right),\left(\varphi, \dot{\varphi}_{0}\right)$ : then the two independent phases and amplitudes are obtained as

$$
\left\{\begin{array}{l}
c^{2}=(c X)^{2}+(c U)^{2}, \quad d^{2}=(d Y)^{2}+(d V)^{2}, \\
\tan a_{0}=c U / c X, \quad \tan b_{0}=d V / d Y .
\end{array}\right.
$$

The remaining quantities are finally determined from Eqs. (A.7)-(A.9).

\section{Appendix B: Potential, gravitational energy an TF components in homeoidal systems}

As in Eqs. (11), (12) and (19) let use assume that

$\rho=\rho_{\mathrm{n}} \tilde{\rho}(m)$,

where $m$ is defined as in Eq. (13), and $\rho_{\mathrm{n}}$ is a normalization constant for density. The total mass of the model (when finite) is given by

$M=4 \pi a_{1} a_{2} a_{3} \rho_{\mathrm{n}} \mathcal{M}$,

where

$\mathcal{M} \equiv \int_{0}^{\infty} \tilde{\rho}(m) m^{2} \mathrm{~d} m$.

For example, in Eqs. (11), (12), and (19) $\mathcal{M}=1 /(3-\gamma)$, $\mathcal{M}=1 / 30$, and $\mathcal{M}=\pi / 4$, respectively. From potential theory, the general expression for $\phi_{\mathrm{g}}(\boldsymbol{\xi})$ is found using the well-known formulas (Kellog 1953; Chendrasekhar 1969; BT87),

$\phi(\boldsymbol{x})=-G \pi \rho_{\mathrm{n}} a_{1} a_{2} a_{3} \int_{0}^{\infty} \frac{\widetilde{\Delta \Psi}[m(\tau, \boldsymbol{x})]}{\Delta(\tau)} \mathrm{d} \tau$

where

$\Delta(\tau)=\sqrt{\left(a_{1}^{2}+\tau\right)\left(a_{2}^{2}+\tau\right)\left(a_{3}^{2}+\tau\right)}$

$\widetilde{\Delta \Psi}(m)=2 \int_{m(\tau, x)}^{\infty} \tilde{\rho}(t) t \mathrm{~d} t$,

and

$m^{2}(\tau, \boldsymbol{x})=\sum_{i=1}^{3} \frac{x_{i}^{2}}{a_{i}^{2}+\tau}$.

For homeoidal density distributions the potential energy tensor components are given by (Roberts 1962)

$U_{i i}=-G \pi^{2} \rho_{\mathrm{n}}^{2} a_{1} a_{2} a_{3} a_{i}^{2} w_{i} \int_{0}^{\infty} \widetilde{\Delta \Psi}^{2}(m) \mathrm{d} m$,

where

$w_{i}=a_{1} a_{2} a_{3} \int_{0}^{\infty} \frac{\mathrm{d} \tau}{\left(a_{i}^{2}+\tau\right) \Delta(\tau)}$ 
and $U=U_{11}+U_{22}+U_{33}$. The three integrals $w_{i}$ are calculated by using identities 238.03, 238.04 and 238.05 from Byrd \& Friedman (1971):

$w_{1}=2 \tilde{a}_{2} \tilde{a}_{3} \frac{F(\varphi, k)-E(\varphi, k)}{k^{2} \sin ^{3} \varphi}$

$w_{2}=2 \tilde{a}_{2} \tilde{a}_{3} \frac{E(\varphi, k)-k^{\prime 2} F(\varphi, k)-k^{2}\left(a_{3} / a_{2}\right) \sin \varphi}{k^{2} k^{\prime 2} \sin ^{3} \varphi}$,

$w_{3}=2 \tilde{a}_{2} \tilde{a}_{3} \frac{\left(a_{2} / a_{3}\right) \sin \varphi-E(\varphi, k)}{k^{\prime 2} \sin ^{3} \varphi}$,

where $\tilde{a}_{2}=a_{2} / a_{1}, \tilde{a}_{3}=a_{3} / a_{1}, \varphi \equiv \arcsin \sqrt{1-\tilde{a}_{3}^{2}}, k^{2} \equiv(1-$ $\left.\tilde{a}_{2}^{2}\right) /\left(1-\tilde{a}_{3}^{2}\right)$, and finally

$$
\left\{\begin{array}{l}
F(\varphi, k)=\int_{0}^{\varphi} \frac{\mathrm{d} t}{\sqrt{1-k^{2} \sin ^{2} t}}, \\
E(\varphi, k)=\int_{0}^{\varphi} \sqrt{1-k^{2} \sin ^{2} t} \mathrm{~d} t,
\end{array}\right.
$$

are the Elliptic Integrals of the first and second kind, respectively and $k^{\prime 2}=1-k^{2}$. Also, we recall that the TF tensor is given by $T_{i j}=-\partial^{2} \phi / \partial x_{i} \partial x_{j}$ : the explicit evaluation of this quantity by using Eq. (B.4) proves Eq. (20). We also evaluate the quantity $2 \pi G \rho_{\mathrm{c} 0}$ needed there by using the virial theorem, $M_{\mathrm{c}} \sigma_{\mathrm{V}}^{2}=-U$. From Eqs. (B.2) and (B.8) we obtain the exact expression

$$
2 \pi G \rho_{\mathrm{n}}=\frac{8 \mathcal{M} \sigma_{\mathrm{V}}^{2}}{\sum_{i=1}^{3} a_{i}^{2} w_{i} \int_{0}^{\infty} \widetilde{\Delta \Psi}^{2}(m) \mathrm{d} m} .
$$

For the density distribution in Eq. (19) it can be proved that the value of the integral at denominator equals $\mathcal{M}=\pi / 4$.

\section{Appendix C: Expansions for small flattenings}

The explicit form of the potential $\phi$ generated by a generic homeoidal density distributions usually cannot be expressed in explicit, closed form. On the contrary, its expression in the limit of small flattenings is trivial. As described in Sect. 3, we introduce the two ellipticities with $a_{2} / a_{1}=1-\epsilon$ and $a_{3} / a_{1}=1-\eta$; obviously, in spherical models $\epsilon=\eta=0$. We now expand Eq. (B.4) for small flattenings, retaining only linear terms. We perform this expansion at fixed mass, i.e, we eliminate the coefficient $\pi a_{1} a_{2} a_{3} \rho_{\mathrm{n}}$ between Eqs. (B.3) and (B.4). With the normalization of lengths to $a_{1}$, a tedious but straightforward calculation shows that $\phi=-\left(G M / a_{1}\right) \tilde{\phi}$, where

$$
\begin{aligned}
\mathcal{M} \times \tilde{\phi}= & \frac{I_{2}(\tilde{r})}{\tilde{r}}+\frac{(3+\epsilon+\eta)\left[I_{1}(\infty)-I_{1}(\tilde{r})\right]}{3} \\
& +\frac{(\epsilon+\eta) I_{4}(\tilde{r})}{3 \tilde{r}^{3}}-\frac{\left(\tilde{y}^{2} \epsilon+\tilde{z}^{2} \eta\right) I_{4}(\tilde{r})}{\tilde{r}^{5}}
\end{aligned}
$$

and $I_{n}(x) \equiv \int_{0}^{x} \tilde{\rho}(t) t^{n} \mathrm{~d} t$. Many dynamical properties of general density-potential pairs obtained with this truncation procedure are presented elsewhere (for a full discussion of this technique see Ciotti \& Bertin 2004).
For example, for ellipsoidal Hernquist models,

$$
\begin{aligned}
& I_{1}(\tilde{r})=\frac{\tilde{r}(2+\tilde{r})}{2(1+\tilde{r})^{2}}, \\
& I_{2}(\tilde{r})=\frac{\tilde{r}^{2}}{2(1+\tilde{r})^{2}}, \\
& I_{4}(\tilde{r})=\frac{\tilde{r}\left(6+9 \tilde{r}+2 \tilde{r}^{2}\right)}{2(1+\tilde{r})^{2}}-3 \ln (1+\tilde{r}) .
\end{aligned}
$$

Note that, at the center of the Hernquist model, the force remains finite but is not continuous, i.e., it depends on the approaching direction, and this fact produces undesidered orbital scattering due to amplification of numerical errors. This problem is avoided when using a density such that in Eq. (12), from which

$I_{1}(\tilde{r})=\frac{\tilde{r}^{2}\left(10+10 \tilde{r}+5 \tilde{r}^{2}+\tilde{r}^{3}\right)}{20(1+\tilde{r})^{5}}$

$I_{2}(\tilde{r})=\frac{\tilde{r}^{3}\left(10+5 \tilde{r}+\tilde{r}^{2}\right)}{30(1+\tilde{r})^{5}}$,

$I_{4}(\tilde{r})=\frac{\tilde{r}^{5}}{5(1+\tilde{r})^{5}}$

Note that the force now vanishes at the model center.

We also expand up to linear terms the three functions in Eqs. (B.10)-(B.12) for $\mu \rightarrow 0$ and $v \rightarrow 0$ :

$$
\begin{aligned}
& w_{1} \simeq 2(1-\mu)(1-v)\left(\frac{1}{3}+\frac{\mu+v}{5}\right), \\
& w_{2} \simeq 2(1-\mu)(1-v)\left(\frac{1}{3}+\frac{3 \mu+v}{5}\right), \\
& w_{3} \simeq 2(1-\mu)(1-v)\left(\frac{1}{3}+\frac{\mu+3 v}{5}\right) .
\end{aligned}
$$

With the aid of these expansions we can finally obtain also the linearization of the gravitational potential energy given in Eq. (B.8)

$U \simeq-\frac{G M^{2}}{a_{1}} \frac{\int_{0}^{\infty} \widetilde{\Delta \Psi}^{2}(m) \mathrm{d} m}{8 \mathcal{M}^{2}}\left(1+\frac{\mu+v}{3}\right)$.

and Eq. (B.14):

$2 \pi G \rho_{\mathrm{c} 0} \simeq \frac{4 \mathcal{M} \sigma_{\mathrm{V}}^{2}}{a_{1}^{2} \int_{0}^{\infty} \widetilde{\Delta \Psi}^{2}(m) \mathrm{d} m}\left[1+\frac{2(\mu+v)}{3}\right]$.

\section{References}

Adams, M. T., Strom, K. E., \& Strom, S. E. 1980, ApJ, 238, 445 Arnaboldi, M., Aguerri, J. A. L., Napolitano, N. R., et al. 2002, AJ, 123,760

Arnaboldi, M., Freeman, K. C., Okamura, S., et al. 2003, AJ, 125, 514 Arnaboldi, M., Gerhard, O., \& Freeman, K. C. 2003, ESO Messenger, 112,37 
Byrd, P. F., \& Friedman, M. D. 1971, Handbook of Elliptic Integrals for Engineers and Scientists (New York: Springer-Verlag)

Chandrasekhar, S. 1969, Ellipsoidal Figures of Equilibrium (New York: Dover)

Ciotti, L., \& Bertin, G. 2004, in preparation

Ciotti, L., \& Dutta, S. N. 1994, MNRAS, 270, 390 (CD94)

Ciotti, L., \& Giampieri, G. 1998, Cel. Mech. \& Dyn. Astron., 68, 313 (CG98)

Durrell, P. R., Ciardullo, R., Feldmeier, J. J., Jacoby, G. H., \& Sigurdson, S. 2002, ApJ, 570, 119

Dehnen, W. 1993, MNRAS, 265, 250

Feldmeier, J. J., Ciardullo, R., \& Jacoby, G. H. 1998, ApJ, 503, 109

Ferguson, H. C., Tanvir, N. R., \& von Hippel, T. 1998, Nature, 391, 461

Ferrers, N. M. 1887, Quart. J. Pure and Appl. Math., 14, 1

Hawley, D. L., \& Peebles, P. J. E. 1975, AJ, 80, 477

Hernquist, L. 1990, ApJ, 356, 359

Kellogg, O. D. 1953, Foundations of Potential Theory (New York: Dover)

Méndez, R. H., Guerrero, M. A., Freeman, K. C., et al. 1997, ApJ, 491, L23

Merritt, D. 1983, ApJ, 264, 24

Merritt, D. 1984, ApJ, 276, 26

Merritt, D. 1985, ApJ, 289, 18
Moore, B., Katz, N., Lake, G., Dressler, A., \& Oemler, A. 1996, Nature, 379, 613

Muccione, V., \& Ciotti, L. 2003, in Computational astrophysics in Italy: methods and tools, Mem. Soc. Astron. Ital. Suppl. (online edition), ed. R. Capuzzo-Dolcetta, 1, 186 (MC03a)

Muccione, V., \& Ciotti, L. 2003, in Galaxies and Chaos, Lecture Notes on Physics, ed. G. Contopoulos, \& N. Voglis (New York: Springer-Verlag), 626, 387 (MC03b)

Napolitano, N. R., Pannella, M., Arnaboldi, M., et al. 2003, ApJ, 594, 172

Nipoti, C., Stiavelli, M., Ciotti, L., Treu, T., \& Rosati, P. 2003, MNRAS, 344, 748

Okamura, S., Yasuda, N., Arnaboldi, M., et al. 2002, PASJ, 54, 883

Press, W. H., Flannery, B. P., Teukolsky, S. A., \& Vetterling, W. T. 1986, Numerical Recipes (Cambridge: Cambridge University Press)

Roberts, P. H. 1962, ApJ, 136, 1108

Schwarzschild, M. 1979, 232, 236

Theuns, T., \& Warren, S. J. 1996, MNRAS, 284, L11

Thompson, L. A. 1976, ApJ, 209, 22

Totani, T. 2003, ApJ, 586, 735

Trevese, D., Cirimele, G., \& Flin, P. 1992, AJ, 104, 935

Valluri, M. 1993, ApJ, 408, 57 